\title{
Prognostic value of legumain in uveal melanoma
}

\author{
TONG WU ${ }^{1}$, LEI SUN ${ }^{2}$, YING WU $^{3}$, RONG XIANG ${ }^{3}$, YUWEI LI $^{4}$, \\ WEINING RONG ${ }^{4}$, FENGYUAN SUN ${ }^{1}$ and NINGLI WANG ${ }^{2}$

\begin{abstract}
${ }^{1}$ Department of Ophthalmology, Tianjin First Central Hospital, Tianjin Medical University, Tianjin 300070; Beijing 100730; ${ }^{3}$ Department of Immunology, Nankai University, Tianjin 300071;

${ }^{4}$ Clinical Medical College, Tianjin Medical University, Tianjin 300070, P.R. China
\end{abstract} \\ ${ }^{2}$ Department of Ophthalmology, Beijing Tongren Eye Center, Beijing Tongren Hospital, Capital Medical University,
}

Received January 16, 2015; Accepted November 5, 2015

DOI: $10.3892 / \mathrm{mmr} .2016 .4838$

\begin{abstract}
The present study aimed to assess the expression of legumain in uveal melanoma (UM) cell lines and primary UM specimens, and to determine the possible association between legumain expression and clinical as well as pathological characteristics to reveal its impact on the prognosis of patients with UM. Records of primary UM cases treated at Beijing Tongren Hospital and Tianjin Eye Hospital between 1996 and 2005 were retrieved for analysis and a total of 82 patients with uveal melanoma were included in the study. The expression of legumain in the formalin-fixed and paraffin-embedded surgical specimens of these 82 patients was determined using immunohistochemical analysis. In addition, the expression of legumain was examined in two uveal melanoma cell lines using polymerase chain reaction and western blot analyses. The association of legumain expression with clinical/pathological characteristics was analyzed using the $\chi^{2}$ and Fisher's exact test. In addition, the impact of legumain on the prognosis of patients with uveal melanoma was examined. Upregulation of legumain was more predominant in the highly invasive uveal melanoma cell line MUM-2B compared with that in the MUM-2C with low invasiveness. Of 82 primary uveal melanoma tissues, 35 exhibited high expression of legumain, while the other 47 specimens exhibited low or negative expression of legumain. High legumain expression was primarily associated with local invasion of UM. Overall survival analysis revealed that the patients with high legumain expression exhibited poorer survival than patients with low/negative legumain expression. These findings suggested that upregulation of legumain is associated with malignant behavior of UM and that legumain may be used as an negative prognostic factor as well as a therapeutic target.
\end{abstract}

Correspondence to: Dr Fengyuan Sun, Department of Ophthalmology, Tianjin First Central Hospital, Tianjin Medical University, 24 Fukang Road, Tianjin 300070, P.R. China

E-mail: fengyuan121new@sina.com

Key words: uveal melanoma, legumain, invasion, prognosis, survival analysis

\section{Introduction}

Uveal melanoma (UM) is the most common type of primary malignant intraocular tumor in adults. Hematogenous dissemination is the predominant avenue for UM metastasis due to the lack of lymphatic vessels in the eyes (1). UM is highly malignant and the primary site of UM metastasis is the liver. Approximately $50 \%$ of patients with UM develop metastasis within 15 years after diagnosis of the primary tumor and the mortality rate is $40-50 \%(2,3)$. Therefore, it is necessary to discover prognostic markers to identify UM cells with high invasive and metastatic potential and to develop therapies to prevent the generation of metastasis from these cells.

Legumain is a cysteine endopeptidase of the asparaginyl endopeptidase family (4), which has been identified in parasites (5) and mammals (6). Overexpression of legumain has been reported in several types of human solid tumor, including colon and breast cancers (7-9). Legumain has a highly restricted specificity requiring an asparagine at the P1 site of its substrates (10). It has also been demonstrated to activate pro-gelatinase $\mathrm{A}$, which is required for extracellular matrix degradation (7,11). Cells overexpressing legumain possess an increased migratory activity in vitro and exhibit invasive and metastatic phenotypes in vivo. Therefore, legumain has been suggested to be involved in tumor invasion and metastasis by degrading extracellular matrix proteins (7). Due to the enrichment of legumain in tumors and its unique restricted specificity, it has been considered to be a potential target for inhibiting tumor metastasis (12-14) and for pro-drug therapy (7,15-17).

However, to the best of our knowledge, the expression of legumain and its impact on the prognosis of patients with UM have not yet been investigated. The purpose of the present study was to determine the expression of legumain in UM cell lines and primary UM specimens, and to determine the association between legumain expression and clinical and pathological characteristics to ultimately reveal its impact on the prognosis of patients with UM.

\section{Materials and methods}

Patients and samples. The records of primary UM cases treated at Beijing Tongren Hospital (Beijing, China) and Tianjin Eye Hospital (Tianjin, China) between 1996 and 2005 were 
retrieved for analysis. The inclusion criteria for the present study were as follows: Histological confirmation of primary UM, no previous history of UM, radiotherapy, thermotherapy or chemotherapy, and the availability of data from a five-year follow-up. A total of 82 cases, comprising 45 males (age, 48-72 years) and 37 females (age, 46-70 years) were enrolled in the present study and all patients had provided written informed consent to their tumor tissues being used for scientific studies. Formalin-fixed and paraffin-embedded surgical specimens of these 82 patients were obtained. The study protocols were approved by the Medical Ethics Committee of Tianjin Eye Hospital (Tianjin, China) and Beijing Tongren Hospital (Beijing, China).

Cell lines and culture. The highly invasive UM cell line MUM-2B and the poorly invasive UM cell line MUM-2C at passage 6 were established by Seftor et al (18) and kindly provided by Dr Rong Xiang (College of Medicine, University of Nankai, Tianjin, China). Cells were incubated in RPMI-1640 medium (Gibco; Thermo Fisher Scientific, Inc., Waltham, MA, USA) containing $10 \%$ fetal bovine serum (Gibco; Thermo Fisher Scientific, Inc.) at $37^{\circ} \mathrm{C}$ in a humidified atmosphere containing $5 \% \mathrm{CO}_{2}$.

Reverse-transcription quantitative polymerase chin reaction $(R T-q P C R)$. Total RNA was isolated from UM cell lines using TRIzol reagent (Invitrogen; Thermo Fisher Scientific, Inc.) and cDNA was synthesized using the Superscript III First Strand Synthesis system (Invitrogen; Thermo Fisher Scientific, Inc.) according to the manufacturer's instructions. PCR was performed using IU Taq DNA Polymerase (Sigma-Aldrich, St. Louis, $\mathrm{MO}$, USA) and $\mathrm{MgCl}_{2}$ at a final concentration of $1.5 \mathrm{mM}$ in a total volume of $25 \mu \mathrm{l}$. qPCR was performed using Fast SYBR Green Master mix, according to the manufacturer's instructions (Applied Biosystems Inc., Foster City, CA, USA) and GAPDH was used as the control gene. The following primers were used: Legumain, forward: 5'-GATGAACCACCTGCCGGATAA-3' and reverse: 5'-CATCATAGTAACAGGCGTAGGACGA-3'; GAPDH, forward: 5'-GAACGGGAAGCTCACTGG-3' and reverse: 5'-TCCACCACCCTGTTGCTGTA-3'. The PCR primers were synthesized by Shanghai Invitrogen Biotechnology Co., Ltd. (Shanghai, China). PCR cycles consisted of initial denaturation at $95^{\circ} \mathrm{C}$ for $5 \mathrm{~min}$ followed by 35 amplification cycles of denaturation at $94^{\circ} \mathrm{C}$ for $45 \mathrm{sec}$, annealing at $50^{\circ} \mathrm{C}$ for $45 \mathrm{sec}$ and extension at $72^{\circ} \mathrm{C}$ for $45 \mathrm{sec}$, and final extension at $72^{\circ} \mathrm{C}$ for $10 \mathrm{~min}$. Each reaction was performed in triplicate. The $2^{-\Delta \Delta \mathrm{Cq}}$ method was used to calculate the relative expression levels of the target gene (19). The expression level of Legumain was log2-transformed and expressed as the legumain/GAPDH expression ratio $\left(2^{-\Delta \Delta \mathrm{CT}}\right)$.

Western blot analysis. Whole-cell protein samples were prepared by homogenization in RIPA lysis buffer (Nanjing KeyGen Biotech Co., Ltd., Nanjing, China) supplemented with a protease and phosphatase inhibitor cocktail. The lysate samples were mixed thoroughly in Eppendorf tubes with equal volumes of electrophoresis sample buffer containing $\beta$-mercaptoethanol (Sigma-Aldrich), and boiled in a thermomixer at $99^{\circ} \mathrm{C}$ for $5 \mathrm{~min}$ at $12,000 \mathrm{x}$. The protein concentration of the lysate was determined using a BCA protein assay kit (Pierce Biotechnology, Inc., Rockford, IL, USA). Equal quantities of protein (40 $\mu \mathrm{g} / \mathrm{lane})$ were separated by $12 \%$ SDS PAGE (Huaxingbio Science and Technology Co., Ltd., Beijing, China). Denatured protein lysate was centrifuged at $12,000 \mathrm{x} \mathrm{g}$ and resolved using gradient (4-20\%) Tris-HCl gels (Bio-Rad Laboratories, Hercules, CA, USA) in electrophoresis buffer (Takara Bio, Inc., Dalian, China) at $200 \mathrm{~V}$ and $350 \mathrm{~mA}$ for $1 \mathrm{~h}$. The separated proteins were immobilized onto polyvinylidene fluoride (PVDF) membranes at $100 \mathrm{~V}$ and $350 \mathrm{~mA}$ for $1 \mathrm{~h}$ according to the manufacturer's instructions. The PVDF membranes were washed five times with phosphate-buffered saline (PBS) containing Tween-20 (Sigma-Aldrich) for $5 \mathrm{~min}$, blocked with blocking buffer (5\% non-fat dry milk) and incubated for $2 \mathrm{~h}$ at room temperature with agitation. Subsequently, the membranes were incubated with rabbit polyclonal anti-legumain antibody (1:1,000 dilution; cat. no. HPA001426; Sigma-Aldrich) for $12 \mathrm{~h}$ at $4^{\circ} \mathrm{C}$. Membranes were then incubated with peroxidase-conjugated goat anti-rabbit immunoglobulin G (1:5,000 dilution; cat. no. A0545; Sigma-Aldrich) in 3\% non-fat dry milk for $2 \mathrm{~h}$ at room temperature with agitation. Between all incubation steps, the membranes were washed five times with PBS containing Tween-20 for $5 \mathrm{~min}$ with agitation. The immunoreactive bands were visualized using ECL Western Blotting Reagents (RPN2106GE; GE Healthcare, Little Chalfont, UK) and visualized on Medical X-ray films (Kodak, Rochester, NY, USA). X-ray films were scanned in a calibrated densitometer (GS-800; Bio-Rad Laboratories Inc., Hercules, CA, USA) and quantified with Image J software (version 1.49; National Institutes of Health, Bethesda, MD, USA). $\beta$-actin (mouse monoclonal antibody; 1:1,000 dilution; cat. no. AA128; Beyotime Institute of Biotechnology, Shanghai, China) was used as a loading control, and its molecular weight ranged from 42 to $43 \mathrm{kDa}$.

Immunofluorescence staining. Melanoma cell lines (30,000 cells/chamber) were grown on cover slips and fixed in $4 \%$ paraformaldehyde for $10 \mathrm{~min}$ at room temperature, rinsed twice with PBS, permeabilized with $0.01 \%$ Triton X-100 (Sigma-Aldrich) in PBS for $10 \mathrm{~min}$ and blocked in $3 \%$ bovine serum albumin (Sigma-Aldrich)/PBS for $1 \mathrm{~h}$. The cells were incubated with 1:1,000 primary antibody (rabbit polyclonal anti-legumain antibody; Sigma-Aldrich) overnight at $4^{\circ} \mathrm{C}$, and subsequent to washing, incubated with secondary antibody (Alexa Fluor $^{\circledR} 488$ Goat Anti-Rabbit SFX kit; cat. no. A31627; 1:1,000 dilution; Invitrogen; Thermo Fisher Scientific, Inc.) at room temperature for $2 \mathrm{~h}$. Finally, the cells were mounted in mounting medium containing 4',6-diamidino-2-phenylindole dihydrochloride (DAPI; Vectashield ${ }^{\circledR}$ Mounting Medium with DAPI; Vector Laboratories, Inc., Burlingame, CA, USA). Fluorescence images were captured and analyzed using a Nikon Eclipse 90i microscope (Nikon Corp., Tokyo, Japan).

Immunohistochemistry. Immunohistochemical staining was performed on $4-\mu \mathrm{m}$ formalin-fixed, paraffin-embedded sections. The sections were incubated at $60^{\circ} \mathrm{C}$ for $12 \mathrm{~h}$ and de-paraffinized 2-3 times in xylene for $10 \mathrm{~min}$ each, followed by incubation in $100 \%$ ethanol for $5 \mathrm{~min}$, hydration in 95 , 70,50 and $30 \%$ ethanol for $5 \mathrm{~min}$ each, and de-pigmentation in kalium hypermanganicum for $30 \mathrm{~min}$. The sections were rinsed in ethanedioic acid and then in PBS three times 


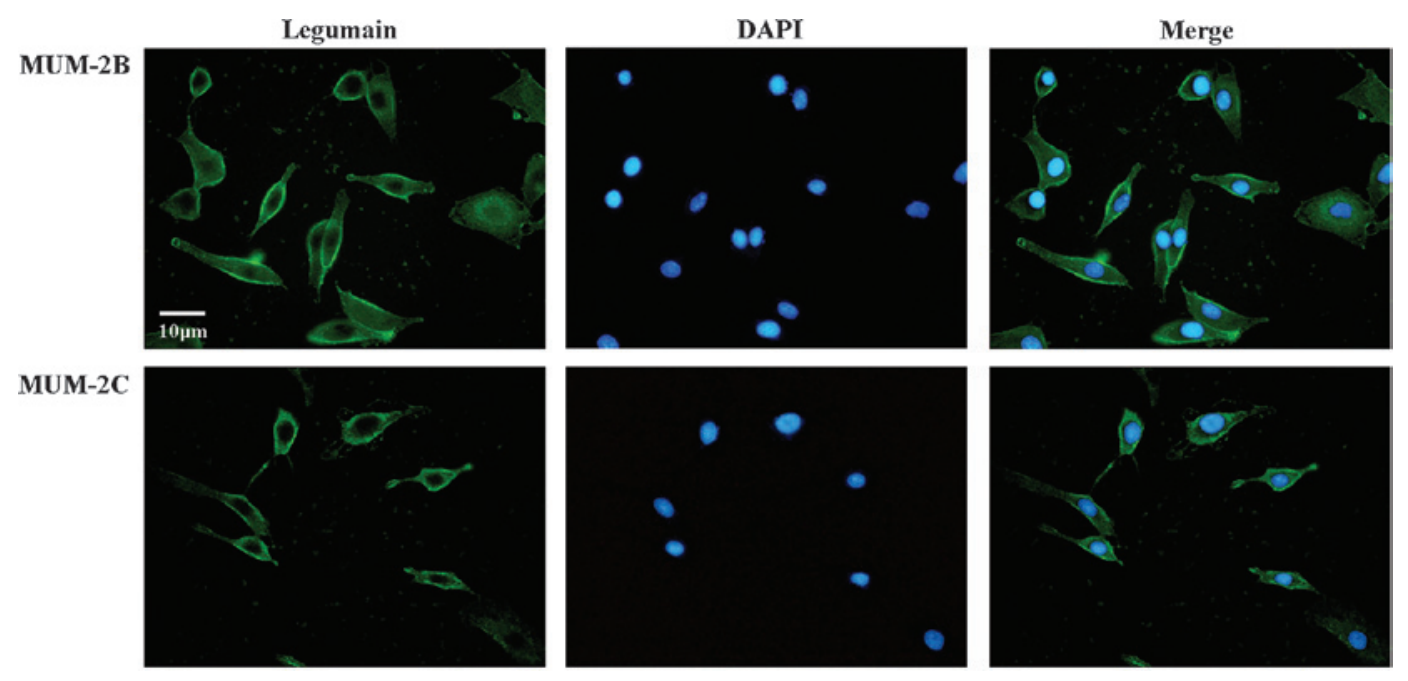

Figure 1. Immunofluorescence images of the MUM-2B and MUM-2C uveal melanoma cell lines (scale bar, $10 \mu \mathrm{m}$ ). Left panel, legumain was expressed in the two uveal melanoma cell lines; middle panel, nuclear staining with DAPI (blue); right panel, merged images.

prior to transfer into citrate buffer. For antigen retrieval, the sections were placed in a high-pressure cooker for $8 \mathrm{~min}$ and then incubated at room temperature for $30 \mathrm{~min}$. The sections were then rinsed in PBS (pH 7.4) and incubated with $3 \%$ $\mathrm{H}_{2} \mathrm{O}_{2}$ in methanol for 20 min to block endogenous peroxidase activity. Non-specific binding of antibody was prevented by pre-incubating the sections with goat serum for $60 \mathrm{~min}$. After removal of the blocking solution, the sections were incubated with rabbit polyclonal anti-legumain antibody (1:500 dilution; Sigma-Aldrich) in 4\% goat serum at room temperature for $1 \mathrm{~h}$. Subsequently, the sections were incubated with horseradish peroxidase (HRP)-conjugated goat anti-rabbit immunoglobulin (1:1,000 dilution; Sigma-Aldrich) for $60 \mathrm{~min}$. The sections were washed with PBS between each incubation step. Antibodies were visualized using staining with a 3,3'-diaminobenzidine tetrahydrochloride substrate kit (Beijing Zhongshan Golden Bridge Biotechnology Co., Ltd., Beijing, China) for $3 \mathrm{~min}$. Next, the sections were rinsed with water, followed by counterstaining with hematoxylin and eosin (Beyotime Institute of Biotechnology). Subsequently, the sections were de-hydrated in 80, 95 and 100\% ethanol for 5 min each, and three times in xylene for 3 min each prior to being mounted.

Histological evaluation of legumain staining in UM specimens. The expression levels of legumain in UM specimens were quantified based on the extent and intensity of the staining. The extent of legumain staining was quantified using the following scale: 0, no staining; 1, staining observed in $\leq 40 \%$ of the viable cells; 2 , staining observed in $>40 \%$ of the viable cells. The intensity of legumain staining was scored as follows: 0, no staining; 1 , low-intensity staining; 2 , high-intensity staining. The specimens were analyzed by two pathologists blinded to the study and the consistency of their scoring was $91 \%$. Whenever differences in the scoring were present, the average of the two score was used as the result.

The total score was defined as the sum of the extent and intensity of legumain staining, and the specimens were divided into two groups based on their total scores: Negative or low legumain expression was defined as a total score of $0-2$, while high legumain expression was defined as a total score of 3-4.
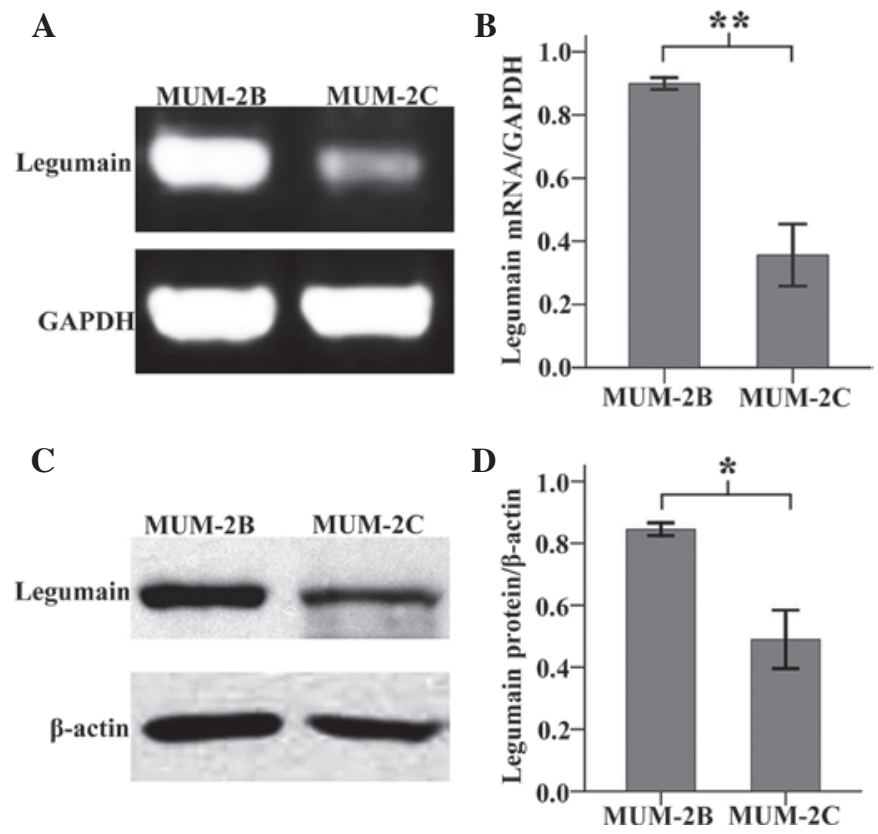

Figure 2. Semi-quantitative analysis of legumain expression in the MUM-2B and MUM-2C uveal melanoma cell lines. (A) Gels showing the polymerase chain reaction amplification products of legumain and GAPDH mRNA in MUM-2B and MUM-2C cells. (B) Quantified expression of legumain mRNA relative to GAPDH in MUM-2B and MUM-2C cells $\left({ }^{* *} \mathrm{P}<0.01 ; \mathrm{n}=3\right.$ for each cell line). The mRNA levels in MUM-2B and MUM-2C cells were $0.89 \pm 0.03$ and $0.37 \pm 0.11$, respectively. (C) Western blot showing the expression of legumain protein in MUM-2B and MUM-2C cells. (D) Quantified expression levels of legumain protein relative to $\beta$-actin in MUM-2B and MUM-2C cells $\left({ }^{*} \mathrm{P}<0.05 ; \mathrm{n}=3\right.$ for MUM-2B and $\mathrm{n}=5$ for MUM-2C). The expression of legumain protein in MUM-2B and MUM-2C cells was $0.82 \pm 0.02$ and $0.48 \pm 0.09$, respectively.

Evaluation of clinical and pathological factors. Pathological analysis of the surgical specimens was performed by two pathologists blinded to the study. The size of the primary tumor was determined by measuring the largest basal diameter (LBD) and the maximum tumor height (MTH). Tumors with $\mathrm{LBD}<10.0 \mathrm{~mm}$ and $\mathrm{MTH}<2.5 \mathrm{~mm}$ were classified as small tumors, while tumors with an $\mathrm{LBD} \geq 10.0 \mathrm{~mm}$ or $\mathrm{MTH} \geq 2.5 \mathrm{~mm}$ were classified as large tumors. Specimens were examined 
Table I. Expression of legumain in the three uveal melanoma cell types.

Legumain expression

\begin{tabular}{lcccr} 
Cell type & Cases, $\mathrm{n}(\%)$ & Low/negative, $\mathrm{n}(\%)$ & High, $(\%)$ & P-value \\
\hline Spindle & $30(36)$ & $24(29)$ & $6(7)$ & 0.004 \\
Mixed & $25(31)$ & $13(16)$ & $12(15)$ & \\
Epithelioid & $27(33)$ & $10(12)$ & $17(21)$ & \\
\hline
\end{tabular}

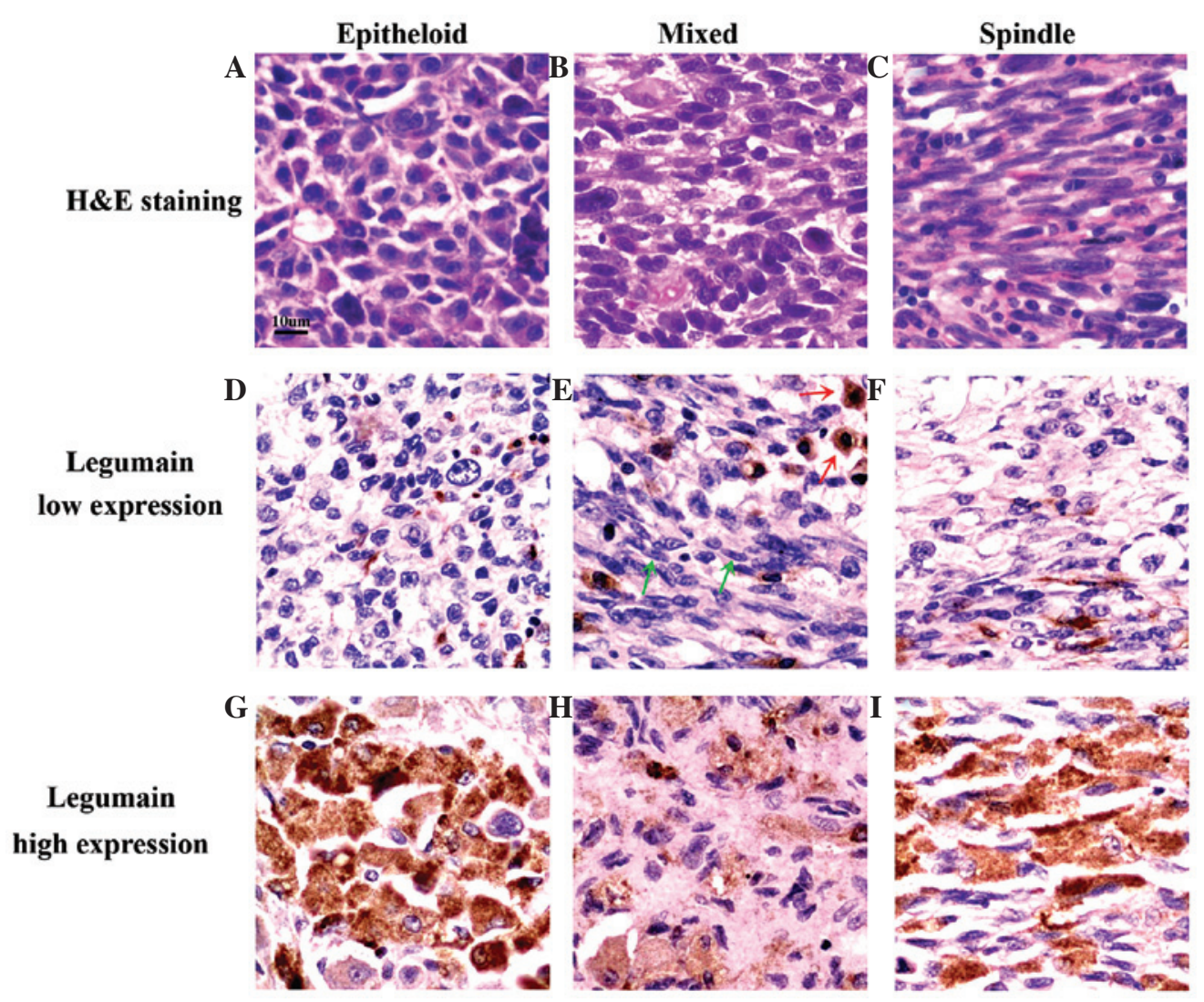

Figure 3. Expression of legumain in three UM cell types. Left panel, H\&E staining showing the morphological classification of the three types of UM cell as (A) epitheloid, (B) mixed and (C) spindle-like. Representative immunohistochemical images of low legumain expression in (D) epithelioid, (E) mixed and (F) spindle-like UM cells. In mixed-cell UM, legumain was preferentially expressed in epithelioid cells (red arrows) rather than in spindle-like cells (green arrows). Representative immunohistochemical images of high legumain expression in (G) epithelioid, (H) mixed and (I) spindle-like UM cells (scale bar, $10 \mu \mathrm{m})$. UM, uveal melanoma; H\&E, hematoxylin and eosin.

for local tumor local invasion. Negative local invasion was defined as the absence of infiltration into the sclera or optic nerve; otherwise, positive local invasion was considered to be present. Metastasis was defined as positive whenever it occurred during a five-year post-operative follow-up period.

Statistical analysis. Values are expressed as the mean \pm the standard deviation. The association of the legumain expression with clinical and pathological features was tested using the $\chi^{2}$ and Fisher's exact test. The association of legumain expression with five-year survival was evaluated using the log-rank test and displayed in Kaplan-Meier plots. A two-tailed $\mathrm{P}<0.05$ was considered to indicate a statistically significant differences. All statistical analyses were performed using SPSS 16.0 (SPSS, Inc., Chicago, IL, USA).

\section{Results}

Legumain expression is upregulated in invasive UM cells. Overexpression of legumain has been observed in several solid tumors and has been indicated to be correlated with tumor invasion and metastasis (7). To investigate whether legumain expression was differentially expressed in invasive or non-invasive UM cells, the MUM-2B and MUM-2C cell lines were subjected to immunofluorescence, western blot and RT-qPCR analyses.

Immunofluorescence staining revealed that legumain was expressed in MUM-2B and MUM-2C cells (Fig. 1). Furthermore, semi-quantitative analysis showed that the expression of legumain mRNA and protein in MUM-2B cells was elevated compared with that in MUM-2C cells (Fig. 2). 
Table II. Association of legumain expression with histological and clinical factors.

\begin{tabular}{lcccr}
\hline & & \multicolumn{2}{c}{ Legumain expression } & \\
\cline { 3 - 4 } Prognostic factor & Cases, $\mathrm{n}(\%)$ & Low/negative, $\mathrm{n}(\%)$ & High, n (\%) & P-value \\
\hline $\begin{array}{l}\text { Tumor size } \\
\text { Small }\end{array}$ & $44(54)$ & $31(38)$ & $13(16)$ & 0.010 \\
Large & $38(46)$ & $16(19)$ & $22(27)$ & \\
Local invasion & & & $1(1)$ & 0.001 \\
$\begin{array}{l}\text { Negative } \\
\text { Positive }\end{array}$ & $28(34)$ & $27(33)$ & $34(41)$ & 0.004 \\
Metastasis & $54(66)$ & $20(25)$ & $23(28)$ & \\
$\quad \begin{array}{l}\text { Negative } \\
\text { Positive }\end{array}$ & $66(80)$ & $43(52)$ & $12(15)$ & \\
\hline
\end{tabular}

Table III. Legumain expression is associated with local invasion in each type of uveal melanoma.

\begin{tabular}{lcccr}
\hline & & \multicolumn{2}{c}{ Legumain expression } & \\
\cline { 3 - 5 } Cell type & Cases, $\mathrm{n}(\%)$ & Low/negative, $\mathrm{n}(\%)$ & High, $\mathrm{n}(\%)$ & P-value \\
\hline Spindle & & & & 0.017 \\
Negative & $15(50)$ & $9(30)$ & $0(0)$ & 0.030 \\
$\begin{array}{l}\text { Positive } \\
\text { Mixed }\end{array}$ & $15(50)$ & $7(28)$ & $6(20)$ & 0.003 \\
Negative & $8(32)$ & $6(24)$ & $1(4)$ & \\
Positive & $17(68)$ & $5(18)$ & $0(0)$ & $17(81)$ \\
Epithelioid & & $5(18)$ & & \\
Negative & $5(18)$ & $22(81)$ & &
\end{tabular}

In Fig 2B, the mRNA levels in MUM-2B and MUM-2C cells were $0.89 \pm 0.03$ and $0.37 \pm 0.11$, respectively. In Fig. $2 \mathrm{D}$, the expression of legumain protein in MUM-2B and MUM-2C cells was $0.82 \pm 0.02$ and $0.48 \pm 0.09$, respectively. Since MUM-2B cells are highly invasive, while MUM-2C display low invasiveness (18), the results suggested that legumain may be a biological marker of invasive UM-cell behavior, and may thus be a potential prognostic factor of UM.

Legumain is differentially expressed among UM-cell types. To investigate the prognostic value of legumain in UM, the expression of legumain was examined in UM specimens by immunohistochemical analysis. Legumain was expressed in most of the UM specimens; based on the total score of legumain staining, 35 of the 82 specimens exhibited high expression, while the other 47 exhibited low expression. The results showed that the expression levels of legumain were independent of age and gender in the high- and low-expression groups, while it was dependent on the type of UM cells.

Within each of the three types of UM cells (epithelioid, mixed or spindle-like) in the tissue specimens, the expression of legumain was found to be either high or low (Fig. 3). However, high expression of legumain was observed to be more common in epithelioid UM cells than in spindle-like UM cells (Table I). In mixed-type UM cells, legumain was preferentially expressed in epithelioid cells (Fig. 3E; red arrows) rather than in spindle-like cells (Fig. 3E; green arrows). The observed preferential expression of legumain in epithelioid cells suggested that legumain may be associated with the enhanced malignant behavior of epitheloid-cell UM (1).

Association of legumain expression with clinical and pathological characteristics. In analogy with other tumor types, the prognosis of UM is affected by more than one factor. Negative clinicopathological prognostic factors in UM include epithelioid-cell morphology, large tumor size as well as the presence of local invasion and metastasis (1). In order to elucidate the role of legumain in the malignant behavior of $\mathrm{UM}$ and assess its prognostic value, it is required to determine the association between the expression of legumain and these clinicopathological factors.

Preliminary analysis showed legumain expression was associated with large tumor size, local invasion and metastasis (Table II). However, tumor size, local invasion and metastasis were all associated with the UM-cell type (data not shown). 
Table IV. Legumain expression is not associated with tumor size or metastasis in each type of uveal melanoma.

\begin{tabular}{|c|c|c|c|c|c|}
\hline \multirow[b]{2}{*}{ Site/cell type } & \multirow[b]{2}{*}{ Size/presence } & \multirow[b]{2}{*}{ Cases, n (\%) } & \multicolumn{2}{|c|}{ Legumain expression } & \multirow[b]{2}{*}{ P-value } \\
\hline & & & Low/negative, n (\%) & High, n (\%) & \\
\hline \multicolumn{6}{|l|}{ Tumor } \\
\hline \multirow[t]{2}{*}{ Spindle } & Small & $23(77)$ & $18(60)$ & $5(17)$ & 1.000 \\
\hline & Large & $7(23)$ & $6(20)$ & $1(3)$ & \\
\hline \multirow{2}{*}{ Mixed } & Small & $15(60)$ & $9(36)$ & $6(24)$ & 0.428 \\
\hline & Large & $10(40)$ & $4(16)$ & $6(24)$ & \\
\hline \multirow[t]{2}{*}{ Epithelioid } & Small & $6(22)$ & $4(15)$ & $2(7)$ & 0.153 \\
\hline & Large & $21(78)$ & $6(22)$ & $15(56)$ & \\
\hline \multicolumn{6}{|l|}{ Metastasis } \\
\hline \multirow[t]{2}{*}{ Spindle } & Negative & $28(94)$ & $23(77)$ & $5(17)$ & 0.366 \\
\hline & Positive & $2(6)$ & $1(3)$ & $1(3)$ & \\
\hline \multirow[t]{2}{*}{ Mixed } & Negative & $19(76)$ & $12(48)$ & $7(28)$ & 0.073 \\
\hline & Positive & $6(24)$ & $1(4)$ & $5(20)$ & \\
\hline \multirow[t]{2}{*}{ Epithelioid } & Negative & $19(70)$ & $8(30)$ & $11(40)$ & 0.666 \\
\hline & Positive & $8(30)$ & $2(7)$ & $6(22)$ & \\
\hline
\end{tabular}

Table V. Legumain expression is not associated with tumor size or metastasis.

Legumain expression

\begin{tabular}{lcccccr}
\cline { 5 - 6 } Site & Local invasion & Size/presence & Cases, $\mathrm{n}(\%)$ & Low/negative, $\mathrm{n}(\%)$ & High, $\mathrm{n}(\%)$ & P-value \\
\hline \multirow{2}{*}{ Tumor } & Negative & Small & $24(86)$ & $24(86)$ & $0(0)$ & 0.143 \\
& & Large & $4(14)$ & $3(11)$ & $1(3)$ & \\
& Positive & Small & $20(37)$ & $7(13)$ & $13(24)$ & 0.812 \\
& & Large & $34(63)$ & $13(24)$ & $21(39)$ & \\
\multirow{3}{*}{ Metastasis } & Negative & Negative & $27(97)$ & $26(94)$ & $1(3)$ & 1.000 \\
& & Positive & $1(3)$ & $1(3)$ & $0(0)$ & \\
& Positive & Negative & $39(72)$ & $17(31)$ & $22(41)$ & 0.108 \\
& & Positive & $15(28)$ & $3(6)$ & $12(22)$ &
\end{tabular}
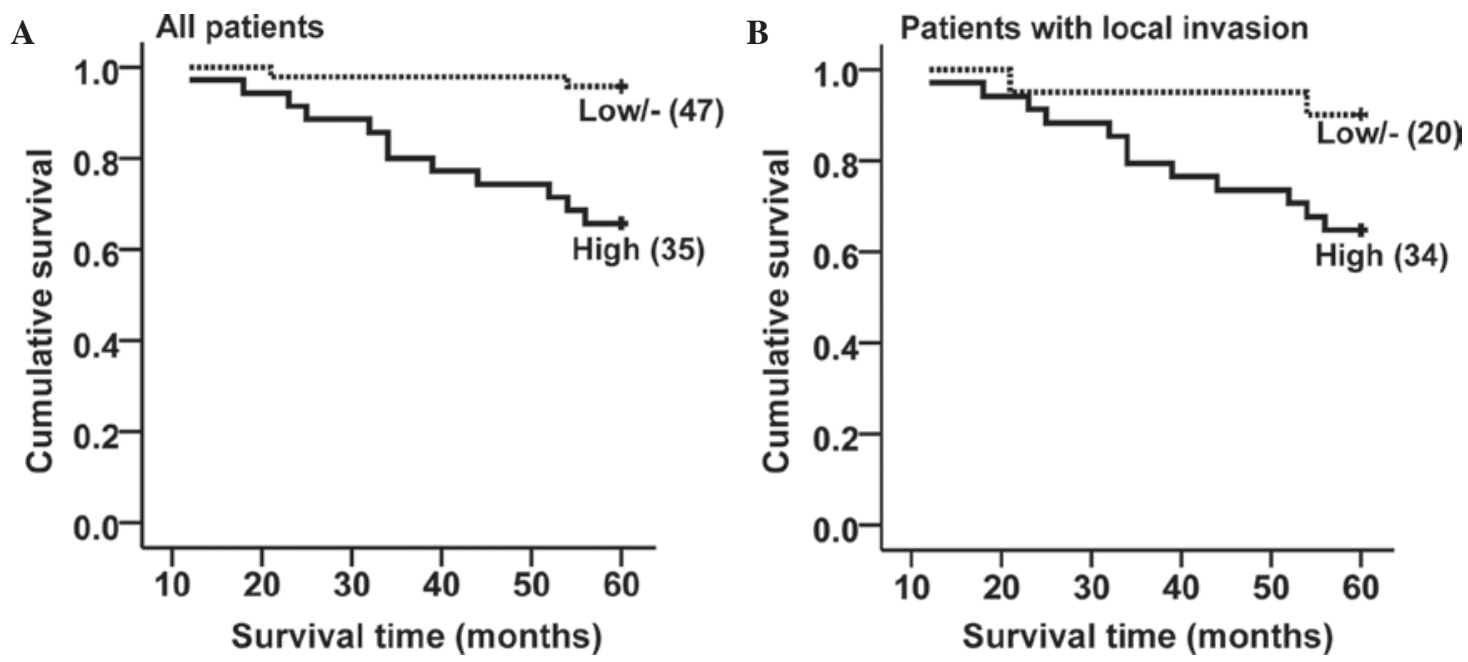

Figure 4. Analysis of the association between legumain expression and prognosis. (A) Kaplan-Meier survival curves of all 82 patients. The patients with high legumain expression exhibited poorer survival as compared to patients with low/negative legumain expression (log-rank test; P<0.001). (B) Kaplan-Meier survival curves of the 54 patients with local invasion. The patients with high legumain expression still exhibited poorer survival as compared to patients with low/negative legumain expression (log rank test, $\mathrm{P}<0.05)$. 
Therefore, legumain expression and prognostic factors may be dependent on the UM-cell type.

To further specify the association of legumain expression with UM tumor behavior, further analysis was performed on each UM-cell type. In contrast to the preliminary results, legumain expression was found to be associated with local invasion (Table III), but not with tumor size or metastasis in every UM-cell type (Table IV). These results suggested that legumain is primarily associated with local invasion of UM.

To confirm that legumain was primarily associated with local invasion, but not with tumor size or metastasis, its expression was compared between tumors with or without local invasion. The results showed that legumain expression was independent of the tumor size or the presence of metastasis, while it was highly asssociated with local invasion (Table V). The association of legumain with tumor size and metastasis shown in Table II is most likely due to its association with local invasion.

High expression of legumain is associated with poor outcome of UM. To determine the prognostic value of legumain in UM, the five-year survival rates were compared between patients with high and those with low/negative legumain expression. The overall survival analysis revealed that the patients with high legumain expression exhibited poorer survival as compared to that of patients with low/negative legumain expression (Fig. 4A; log-rank test, $\mathrm{P}<0.001$ ). Since legumain expression was shown to be associated with local invasion, the negative impact of legumain expression on patient survival is likely to be due to legumain-induced local invasion of UM.

To test whether legumain expression alone had any impact on UM prognosis, further survival analysis was performed in patients with or without local invasion. Among the 28 patients without local invasion, there was only one case of high legumain expression, and all patients had survived at five years following surgery; therefore, it was not possible to perform any survival analysis. Among the 54 patients with local invasion, patients with high legumain expression exhibited poorer survival than patients with low/negative legumain expression (Fig. 4B; log-rank test, $\mathrm{P}<0.046$ ). These results indicated that high legumain expression is a negative prognostic factor for UM.

\section{Discussion}

The prognosis of UM is largely dependent on tumor behavior, which is essentially determined by the tumor-cell type. The UM cell line MUM-2B is highly invasive, while MUM-2C exhibits low invasiveness (18); furthermore, patients diagnosed with epithelioid-cell UM exhibit poorer survival than patients with spindle-cell UM due to the increased invasiveness of the former $(1,3)$. In the present study, legumain was shown to be expressed in MUM-2B as well as MUM-2C, while it was significantly higher in the more invasive MUM-2B cell line compared to that in MUM-2C. Furthermore, legumain expression was observed to be higher in epitheloid-cell UM specimens compared to that in spindle-like UM cells. These results supported the hypothesis that upregulation of legumain is associated with malignant behavior of UM.
While a preliminary experiment indicated that high expression of legumain was associated with large tumor size and the presence of metastasis, it was subsequently revealed that this observation was due to the sole association of legumain with local invasion, while it was independent of tumor size and metastasis. Considering the general mechanism of tumor progression, legumain expression may have a causal association with metastasis by promoting UM metastasis via enhancing the local invasion of UM cells. While no causal association was present between large tumor size and high legumain expression, the likelihood of local invasion may have been increased in large-size tumors, which was promoted by legumain in parallel.

The results of the present study, combined with the results of a previous study (20), indicated that the negative impact of legumain on the prognosis of UM may be attributed to its effect of promoting local invasion. However, survival analysis of the 54 patients with local invasion showed that, by contrast to patients with low/negative legumain expression, high legumain expression was associated with poor survival of UM patients. Since in patients with local invasion, legumain expression was not associated with tumor size or metastasis, it is indicated that legumain may exert its negative effects on UM prognosis through other pathways.

Little is known regarding other biological functions of legumain. A previous study showed that legumain is involved in nucleoplasmic calcium signaling pathways that regulate cell proliferation, and it was suggested that increased expression of legumain might be involved in carcinogenesis (21). Legumain was also shown to be highly expressed in tumor-associated macrophages (TAMs) in the tumor microenvironment (12). TAMs have an important role in tumor angiogenesis and metastasis (22); however, the possible implication of legumain in this process remains elusive. A recent study reported that legumain induced chemotaxis of primary human monocytes and human umbilical vein endothelial cells (23), suggesting that legumain may also have a role in recruiting macrophages and angiogenesis.

Legumain has been shown to be present not only intracellularly in endosome/lysosome systems, but also on the surface of tumor cells and endothelial cells, as well as in the extracellular matrix $(7,16)$. The enrichment of legumain in tumor microenvironments provides opportunities for several potential therapeutic approaces. A novel legumain-activated, cell-impermeable doxorubicin pro-drug, LEG-3, was shown to completely arrest the growth of a variety of neoplasms, including multidrug-resistant tumors, in vivo, and significantly prolonged survival without evidence of myelosuppression or cardiac toxicity (16). Several other novel legumain-activated pro-drugs have also shown promising potency against tumors (15). The enrichment of legumain in the tumor microenvironment also makes it a target for tumor immunotherapy. Studies have shown that a DNA vaccine targeting legumain-expressing cells suppressed tumor growth and dissemination by inducing a specific CD8+ T-cell response against legumain-expressing cells in vivo $(9,12,13)$. Due to the importance of legumain in the promotion of tumor invasion, the suppression of legumain expression or inhibition of its function may also be feasible approaches for anti-tumor therapies. 
The present study revealed that legumain has a negative impact on the prognosis of patients with UM. Patients with UM tissues exhibiting high and extensive staining of legumain exhibited shorter survival than those with weak and confined legumain staining. However, only the combination of high intensity and extent of legumain expression had a significant negative impact on patient survival, suggesting that both factors should be considered when posing a prognosis for UM patients.

The present study indicated that legumain was overexpressed in epithelioid UM cells with high potential of invasion. Legumain was revealed to be a prognostic biomarker for UM with a significant association with local invasion, which requires further clinical evaluation. Furthermore, legumain-activated pro-drugs and anti-legumain immunotherapy may represent novel approaches for UM therapy.

\section{Acknowledgements}

The authors would like to thank Dr Rong Xiang (College of Medicine, University of Nankai, Tianjin, China) for kindly providing the UM cell lines, and Tianjin Eye Hospital (Tianjin, China) and Beijing Tongren Hospital (Beijing, China) for providing the patient samples and data. This study was supported by grants from the National Natural Science Foundation of China (grant no. NSFC 81441027).

\section{References}

1. Mudhar HS, Parsons MA, Sisley K, Rundle P, Singh A and Rennie IG: A critical appraisal of the prognostic and predictive factors for uveal malignant melanoma. Histopathology 45: 1-12, 2004.

2. Singh AD and Borden EC: Metastatic uveal melanoma. Ophthalmol Clin North Am 18: 143-150, 2005.

3. Desjardins L, Levy-Gabriel C, Lumbroso-Lerouic L, Sastre X, Dendale R, Couturier J, Piperno-Neumann S, Dorval T, Mariani P, Salmon R, et al: Prognostic factors for malignant uveal melanoma. Retrospective study on 2,241 patients and recent contribution of monosomy-3 research. J Fr Ophtalmol 29 : 741-749, 2006 (In French).

4. Kembhavi AA, Buttle DJ, Knight CG and Barrett AJ: The two cysteine endopeptidases of legume seeds: Purification and characterization by use of specific fluorometric assays. Arch Biochem Biophys 303: 208-213, 1993.

5. Dalton JP, Hola-Jamriska L and Brindley PJ: Asparaginyl endopeptidase activity in adult Schistosoma mansoni. Parasitology 111: 575-580, 1995.

6. Chen JM, Dando PM, Rawlings ND, Brown MA, Young NE, Stevens RA, Hewitt E, Watts C and Barrett AJ: Cloning, isolation and characterization of mammalian legumain, an asparaginyl endopeptidase. J Biol Chem 272: 8090-8098, 1997.
7. Liu C, Sun C, Huang H, Janda K and Edgington T: Overexpression of legumain in tumors is significant for invasion/metastasis and a candidate enzymatic target for prodrug therapy. Cancer Res 63: 2957-2964, 2003

8. Murthy RV, Arbman G, Gao J, Roodman GD and Sun XF: Legumain expression in relation to clinicopathologic and biological variables in colorectal cancer. Clin Cancer Res 11: 2293-2299, 2005.

9. Gawenda J, Traub F, Lück HJ, Kreipe H and von Wasielewski R: Legumain expression as a prognostic factor in breast cancer patients. Breast Cancer Res Treat 102: 1-6, 2007.

10. Barrett AJ and Rawlings ND: Evolutionary lines of cysteine peptidases. Biol Chem 382: 727-733, 2001.

11. Chen JM, Fortunato M, Stevens RA and Barrett AJ: Activation of progelatinase A by mammalian legumain, a recently discovered cysteine proteinase. Biol Chem 382: 777-783, 2001.

12. Luo Y, Zhou H, Krueger J, Kaplan C, Lee SH, Dolman C, Markowitz D, Wu W, Liu C, Reisfeld RA and Xiang R: Targeting tumor-associated macrophages as a novel strategy against breast cancer. J Clin Invest 116: 2132-2141, 2006.

13. Lewēn S, Zhou H, Hu HD, Cheng T, Markowitz D, Reisfeld RA, Xiang R and Luo Y: A legumain-based minigene vaccine targets the tumor stroma and suppresses breast cancer growth and angiogenesis. Cancer Immunol Immunother 57: 507-515, 2008.

14. Xiang R, Luo Y, Niethammer AG and Reisfeld RA: Oral DNA vaccines target the tumor vasculature and microenvironment and suppress tumor growth and metastasis. Immunol Rev 222: 117-128, 2008.

15. Bajjuri KM, Liu Y, Liu C and Sinha SC: The legumain protease-activated auristatin prodrugs suppress tumor growth and metastasis without toxicity. Chem Med Chem 6: 54-59, 2011.

16. Wu W, Luo Y, Sun C, et al: Targeting cell-impermeable prodrug activation to tumor microenvironment eradicates multiple drug-resistant neoplasms. Cancer Res 66: 970-980, 2006.

17. Stern L, Perry R, Ofek P, Many A, Shabat D and Satchi-Fainaro R: A novel antitumor prodrug platform designed to be cleaved by the endoprotease legumain. Bioconjug Chem 20: 500-510, 2009.

18. Seftor EA, Meltzer PS, Kirschmann DA, Pe'er J, Maniotis AJ, Trent JM, Folberg R and Hendrix MJ: Molecular determinants of human uveal melanoma invasion and metastasis. Clin Exp Metastasis 19: 233-246, 2002.

19. Livak KJ and Schmittgen TD: Analysis of relative gene expression data using real-time quantitative PCR and the 2(-Delta Delta C(T)) Method. Methods 25: 402-408, 2001.

20. Briggs JJ, Haugen MH, Johansen HT, Riker AI, Abrahamson M, Fodstad $\varnothing$, Maelandsmo GM and Solberg R: Cystatin E/M suppresses legumain activity and invasion of human melanoma. BMC Cancer 10: 17, 2010.

21. Andrade V, Guerra M, Jardim C, Melo F, Silva W, Ortega JM, Robert M, Nathanson MH and Leite F: Nucleoplasmic calcium regulates cell proliferation through legumain. J Hepatol 55: 626-635, 2011.

22. Bingle L, Brown NJ and Lewis CE: The role of tumour-associated macrophages in tumour progression: Implications for new anticancer therapies. J Pathol 196: 254-265, 2002.

23. Clerin V, Shih HH, Deng N, Hebert G, Resmini C, Shields KM, Feldman JL, Winkler A, Albert L, Maganti V, et al: Expression of the cysteine protease legumain in vascular lesions and functional implications in atherogenesis. Atherosclerosis 201: 53-66, 2008 . 